\title{
Comparative Assessment of Ergonomic Experience with Heads-Up Display and Conventional Surgical Microscope in the Operating Room
}

This article was published in the following Dove Press journal:

Clinical Ophthalmology

\author{
Robert J Weinstock' \\ Margaret H Ainslie-Garcia ${ }^{2}$ \\ Nicole C Ferko ${ }^{2}$ \\ Rana A Qadeer ${ }^{2}$ \\ Leighton P Morris ${ }^{3}$ \\ Hang Cheng ${ }^{3}$ \\ Justis $P$ Ehlers ${ }^{4,5}$
}

'Eye Institute of West Florida, Largo, FL, USA; ' ${ }^{2}$ Eversana, Burlington, ON, Canada; ${ }^{3}$ Alcon Vision LLC, Fort Worth, TX, USA; ${ }^{4}$ Tony and Leona Campane Center for Excellence in Image-Guided Surgery and Advanced Imaging Research, Cleveland Clinic, Cleveland, OH, USA; ${ }^{5}$ Vitreoretinal Service, Cole Eye Institute, Cleveland, OH, USA
Correspondence: Justis P Ehlers Cole Eye Institute, 9500 Euclid Ave//32, Cleveland, $\mathrm{OH}$, USA

$\mathrm{Tel}+$ I 216-636-0183

Email ehlersj@ccf.org
Purpose: Musculoskeletal pain issues are prevalent in ophthalmic surgeons and can impact surgeon well-being and productivity. Heads-up displays (HUD) can improve upon conventional microscopes by reducing ergonomic stress. This study compared ergonomic outcomes between HUD and a conventional optical microscope in the operating room, as reported by ophthalmic surgeons in the US.

Methods: An online questionnaire was distributed to a sample of surgeons who had experience operating with HUD. The questionnaire captured surgeon-specific variables, the validated Nordic Musculoskeletal Questionnaire, and custom questions to compare HUD and conventional microscope. A multivariable model was built to identify variables that were likely to predict improvement in pain-related issues.

Results: Analysis was conducted on 64 surgeons (37 posterior-segment, 25 anteriorsegment, and two mixed) with a mean 14.9 years of practice and 2.3 years using HUD. Most surgeons agreed or strongly agreed that HUD reduced the severity (64\%) and frequency (63\%) of pain and discomfort, improved posture (73\%), and improved overall comfort (77\%). Of respondents who experienced headaches, or pain and discomfort during operation, $12(44 \%)$ reported their headaches improved and 45 (82\%) reported feeling less pain and discomfort since they started using HUD. The multivariable model indicated the odds of reporting an improvement in pain since introducing the HUD in the operating room were 5.12-times greater for those who used HUD in $>50 \%$ of their cases $(P=0.029)$.

Conclusion: This study indicates that heads-up display may be an important tool for wellness in the operating room as it can benefit ophthalmic surgeons across several ergonomic measures.

Keywords: heads-up display, microscope, ergonomic, musculoskeletal disorders, ophthalmology, surgery

\section{Introduction}

Work-related musculoskeletal disorders (MSDs) accounted for an estimated $31 \%$ of all worker injury and illness cases in the US in 2015. ${ }^{1}$ Several job factors in ophthalmology may contribute to MSDs, including task repetition, working in nonneutral positions (eg, cramped, hyperextended, or twisted), and holding positions for extended periods. Compared to family medicine practitioners, eye care providers had a statistically significantly higher prevalence of pain in the neck, lower back, and hand/wrist areas. ${ }^{2}$ 
The operating room (OR) is an important contributor to MSDs due to physical strain. ${ }^{3,4}$ In particular, poor posture from microscope use can cause ergonomic strain due to suboptimal positions of the neck, ${ }^{5}$ and has been associated with neck and back pain in several surgical specialties. ${ }^{6,7}$

MSDs can have important consequences for the wellbeing of ophthalmic surgeons. A survey of 518 ophthalmologists in the UK reported that $57.1 \%$ used anti-inflammatory drugs, $40 \%$ required physiotherapy, and $5 \%$ had undergone surgery for low back pain. ${ }^{4}$ Productivity is also impaired, with a small number of ophthalmologists reporting MSDs that limited or prevented their ability to operate. ${ }^{3,8}$ A systematic literature review of ergonomic studies across surgical specialties reported that $30 \%$ of surgeons have changed their operative procedures in consideration of their own physical symptoms, ${ }^{9}$ indicating consequences of MSDs may extend to the patient.

Ergonomic interventions in the OR can provide surgeon benefit, as shown with intraoperative microbreaks and monitor use. ${ }^{10,11}$ In the last decade, heads-up digital visualization systems have become available for ophthalmic surgeries and have shown ergonomic benefits ${ }^{12}$ including improved neck and back comfort. ${ }^{13,14}$ However, these studies were limited by small sample size ( $\leq 20$ surgeons) and design (eg, performed tasks on objects or animal models), which are less reflective of the real-world OR environment. In addition, the majority of studies were conducted on posterior-segment surgeons as opposed to anterior-segment surgeons, which underrepresents a considerable portion of ophthalmic surgeons. The purpose of this study was to understand ophthalmic surgeons' use and perceptions of heads-up display (HUD) in the OR, and compare the ergonomic benefits between HUD and a conventional surgical microscope.

\section{Methods}

\section{Study Population and Recruitment}

The study protocol was reviewed by a centralized Institutional Review Board (Sterling IRB) and received exemption status, determining that it did not constitute human subjects research as defined by the Department of Health and Human Services (DHHS 45 CFR 46.102d) or the Food and Drug Administration (FDA 21 CFR 50.3c,56.102c; FDA 21 CFR 812.3p). Ophthalmic surgeons practicing in the US were contacted via email, phone, and mail to participate in this cross-sectional, single-blind study (the data analyst was masked). All surgeons who passed residency, performed ophthalmic surgery, had access to an OR with HUD, and practiced in the US were eligible to participate. A third-party research agency handled recruitment and screening, starting with a list of surgeons at sites where HUD units (ie, NGENUITY ${ }^{\circledR}$ or TrueVision ${ }^{\circledR}$ ) had been installed, provided by the current manufacturer (Alcon Vision LLC, TX). Primary investigators RW and JE provided contact information of surgeons who were less likely to be captured by primary recruitment (ie, those who used alternative HUD devices or were known to have previously used HUD but did not have one installed at their current practice). Participants were aware it was an industrysponsored study, but primary investigators and the manufacturer were masked to the identity of participants. Informed consent was obtained from all participants. Upon completion of the questionnaire, participants received an honorarium to compensate their time appropriately. All respondents could refer additional surgeons to the recruitment agency for screening, without incentive.

\section{Questionnaire}

A literature review of surgical ergonomics informed development of the online questionnaire, which was piloted by RW and JE (neither responses were included in the study cohort). For the complete questionnaire, see the Supplementary Appendix. Part one collected participant demographics (eg, age, years of practice, specialty), musculoskeletal health history (eg, baseline headache and pain using a 10-point visual analog scale), surgical variables (eg, case volume, average case length), and HUD use (eg, years of use, proportion of cases used). In part two, an electronic version of the validated Nordic Musculoskeletal Questionnaire $^{15}$ asked whether participants experienced pain-related issues (defined as "trouble", ie, ache, pain, discomfort, numbness) in the neck, upper back, lower back, or shoulders in the past 12 months. Part three used custom questions to compare the surgeon's perceptions of headache, pain, benefit, and preference between HUD and conventional ocular microscope.

\section{Statistical Analysis}

Population characteristics and questionnaire responses were summarized descriptively for the complete sample. A subgroup analysis comparing anterior- and posteriorsegment surgeons was conducted to determine whether results differed between surgeon types (improvement in headache, pain while operating, pain-related issues, statements of agreement), excluding surgeons who reported 
completing both types of surgery. Characteristics and descriptive frequency distributions were compared using Chi-square and Wilcoxon tests. A multivariable regression analysis was performed to explore variables that may predict improvement in pain-related issues. Recommendations from primary investigators informed variables to be tested (age, cumulative number of operating hours, type of surgeon [anterior/posterior], proportion of cases completed with HUD $[\leq 50 \%$ vs $>50 \%])$. The strongest subset of predictors was selected in a stepwise manner (backwards, forwards, and both) based on the Akaike information criterion. A $P$-value of less than 0.05 was considered statistically significant. Statistical analysis was performed in R, version 3.6.1 (RStudio, Vienna, Austria). ${ }^{16}$

\section{Results}

\section{Population Characteristics}

The survey was completed by 64 surgeons (response rate $42.4 \%)$, including $59(92.2 \%)$ attending surgeons and five (7.8\%) fellows. Surgeons completed posterior-segment surgery only $(37,57.8 \%)$, anterior-segment surgery only $(25,39.1 \%)$ with two $(3.1 \%)$ respondents reporting experience in both areas. Of the 27 anterior-segment surgeons, all performed cataract surgeries, 15 performed minimally invasive glaucoma surgery, and seven performed corneal surgery. Population characteristics including baseline pain and OR variables are summarized in Table 1.

\section{HUD Utilization and Preference}

The NGENUITY ${ }^{\circledR} 3 \mathrm{D}$ visualization system (Alcon Vision LLC) was used by 58 (90.6\%) surgeons, three (4.7\%) used TrueVision $^{\circledR}$ 3D visualization system (TrueVision 3D Systems Inc., CA), two (3.1\%) used both NGENUITY ${ }^{\circledR}$ and TrueVision ${ }^{\circledR}$, and one (1.6\%) used Zeiss Artevo (Zeiss Meditec, Jena, Germany). Mean duration of use for HUD in the sample was 2.27 years $(\mathrm{SD}=1.77$; range $=0.17-10$ years). Eleven surgeons (17\%) reported using HUD for $100 \%$ of cases, while six surgeons $(9 \%)$ reported using the device in $<10 \%$ of cases.

Overall, 44 (69\%) surgeons reported they preferred HUD to microscope, and $55(86 \%)$ would recommend it to their peers. Of the surgeons who preferred HUD, the most common reasons provided were visualization $(77 \%)$, ergonomics and comfort $(61 \%)$, and teaching benefit $(30 \%$ of those at a teaching facility or in a learning role) (Table 2). A conventional microscope was preferred by 20 (31\%) surgeons, with visualization (particularly of the periphery; $42 \%$ ), speed (15\%), and familiarity (12\%) among the top reasons provided (Table 2 ).

\section{Ergonomics and Pain in the OR}

Some respondents were pain-free in some regard, as 58\%, $14 \%$, and $17 \%$ of the sample reported that headaches, pain/ discomfort during operation, or pain-related issues, respectively, were not applicable to them (Figure 1). Of applicable surgeons, 12 (44\%) reported their headaches improved and $45(82 \%)$ reported feeling less pain/discomfort during operation since introducing HUD. Similarly, 40 (75\%) surgeons reported improvement in pain-related issues compared to a time when they only used a microscope (Figure 1).

Of 64 surgeons, the majority agreed or strongly agreed that HUD provided a benefit compared to a conventional microscope in relation to reduced severity (64\%) and frequency of pain $(63 \%)$, and improved posture $(73 \%)$, physical performance (eg, fatigue, stamina, mobility; $58 \%$ ), or overall comfort $(77 \%)$. The majority also agreed or strongly agreed that HUD allowed them to better visualize the areas and angles required for their procedure (64\%), and operate more comfortably under higher magnification (69\%). Most surgeons were neutral about HUD improving mental performance (39\% agreed or strongly agreed) or improving their confidence in ability to perform complex procedures (36\% agreed or strongly agreed).

Among only anterior-segment surgeons, the strongest agreement was seen when comparing HUD to a conventional microscope for reduced severity or frequency of pain and discomfort (Figure 2A and B), improved posture (Figure 2C), and improved overall comfort (Figure 2D), 84\% agreed or strongly agreed for each. Among only posteriorsegment surgeons, the benefit noted by the highest number of participants was improved overall comfort (70\% agreed or strongly agreed, Figure 3D), improved comfort when operating under higher magnification (70\%, Figure 3I), improved posture $(68 \%$, Figure $3 \mathrm{C})$, and better visualization of areas and angles required for surgery $(62 \%$, Figure $3 \mathrm{H})$.

\section{Anterior- versus Posterior-Segment Surgeons}

An exploratory subgroup analysis compared anterior- and posterior-segment surgeons to determine if there were differences in their characteristics or reported benefits of HUD. Characteristics that differed significantly between groups were operating position, average case length, and 
Table I Population Characteristics

\begin{tabular}{|c|c|c|c|}
\hline Characteristics & Total $(n=64)$ & Anterior $(n=25)$ & Posterior $(n=37)$ \\
\hline \multicolumn{4}{|l|}{ Sex } \\
\hline Female (n, \%) & $8(12.5 \%)$ & $5(20.0 \%)$ & $3(8.1 \%)$ \\
\hline Male $(n, \%)^{a}$ & $55(85.9 \%)$ & $20(80.0 \%)$ & $33(89.2 \%)$ \\
\hline Refused to answer (n, \%) & $\mathrm{I}(1.6 \%)$ & $0(0.0 \%)$ & I (2.7\%) \\
\hline Height in inches (mean, SD) & $70.30(4.70)$ & $69.56(3.79)$ & $70.92(5.27)$ \\
\hline Weight in pounds (mean, SD) & $173.56(25.10)$ & $171.64(25.68)$ & I $75.57(25.33)$ \\
\hline Age (mean, SD) & $45.55(9.79)$ & $46.56(10.48)$ & $45.35(9.38)$ \\
\hline Years practicing ophthalmology (mean, SD) & I $4.88(9.44)$ & $16.72(9.98)$ & $14.00(9.14)$ \\
\hline Years using HUD (mean, SD) & $2.27(1.77)$ & $2.20(2.47)$ & $2.27(1.17)$ \\
\hline \multicolumn{4}{|l|}{ Operating room variables } \\
\hline \multicolumn{4}{|l|}{ Operating position (n, \%) } \\
\hline Temporal in $>90 \%$ of cases & 21 (32.8\%) & $2 \mathrm{I}(84.0 \%)^{* *}$ & $0(0.0 \%)^{* *}$ \\
\hline Superior in $>90 \%$ of cases ${ }^{a}$ & $40(62.5 \%)$ & $2(8.0 \%)^{* *}$ & $36(97.3 \%)^{* *}$ \\
\hline Mixed distribution & $3(4.7 \%)$ & $2(8.0 \%)^{* *}$ & I $(2.7 \%)^{* *}$ \\
\hline \multicolumn{4}{|l|}{ Type of Microscope (n, \%) } \\
\hline Floor mounted ${ }^{\mathrm{a}}$ & $55(85.9 \%)$ & $22(88.0 \%)$ & $31(83.8 \%)$ \\
\hline Ceiling mounted & $9(14.1 \%)$ & $3(12.0 \%)$ & $6(16.2 \%)$ \\
\hline Average case length, minutes (mean, SD) & 33.91 (22.29) & II $.80(5.49)^{* *}$ & $47.43(15.30)^{* *}$ \\
\hline Estimated annual case load (mean, SD) & $490.70(467.43)$ & $884.00(539.04)^{* *}$ & $239.05(110.99)^{* *}$ \\
\hline \multicolumn{4}{|l|}{ Estimated proportion of cases completed with HUD $(n, \%)^{b}$} \\
\hline $\mathrm{I}-25 \%{ }^{\mathrm{a}}$ & $15(23.4 \%)$ & $6(24.0 \%)$ & $7(18.9 \%)$ \\
\hline $26-50 \%$ & $16(25.0 \%)$ & $7(28.0 \%)$ & 9 (24.3\%) \\
\hline $51-75 \%$ & 7 (10.9\%) & $3(12.0 \%)$ & $4(10.8 \%)$ \\
\hline $76-100 \%$ & $25(39.1 \%)$ & $9(36.0 \%)$ & $16(43.2 \%)$ \\
\hline \multicolumn{4}{|l|}{ Baseline Pain Characteristics } \\
\hline Headache severity (median, IQR) ${ }^{c}$ & $1.00(0-3.00)$ & $1.00(0.00-4.00)$ & $1.00(0.00-2.00)$ \\
\hline Level of neck or back pain/discomfort ${ }^{\mathrm{d}}$ (median, IQR) & $2.00(1.00-3.00)$ & $3.00(1.00-5.00)^{*}$ & $1.00(1.00-2.00)^{*}$ \\
\hline \multicolumn{4}{|l|}{ Experienced pain-related issues (past 12 months) } \\
\hline Neck ( $\mathrm{n}$ surgeons, \%) ${ }^{\mathrm{a}}$ & 31 (48.4\%) & $10(40.0 \%)$ & 19 (5I.4\%) \\
\hline Upper Back (n, \%) & $20(31.2 \%)$ & $8(32.0 \%)$ & $10(27.0 \%)$ \\
\hline Lower Back (n, \%) ${ }^{a}$ & $26(40.6 \%)$ & $13(52.0 \%)$ & $12(32.4 \%)$ \\
\hline Shoulder (right) $(\mathrm{n}, \%)^{\mathrm{a}}$ & $\mathrm{II}(\mathrm{I} 7.2 \%)$ & $2(8.0 \%)$ & $8(21.6 \%)$ \\
\hline Shoulder (left) (n, \%) ${ }^{a}$ & II (17.2\%) & $3(12.0 \%)$ & 7 (18.9\%) \\
\hline None of the above $(n, \%)$ & 20 (31.2\%) & $6(24.0 \%)$ & 14 (37.8\%) \\
\hline
\end{tabular}

Notes: $* P<0.05$, difference between anterior- and posterior-segment surgeons; $* * P<0.001$, difference between anterior- and posterior-segment surgeons; ${ }^{a}$ Numbers do not sum to total due to respondents that reported performing both anterior- and posterior-segment surgeries; ${ }^{b}$ Missing for one respondent (percentages presented from full sample, $n=64)$; 'Surgeons were asked to rank their average headache severity on a scale of $0-10(0=$ no headaches, $5=$ moderate headaches, and $10=$ worst possible headache); 'Surgeons were asked to rank their average level of neck or back pain/discomfort on a scale of $0-10(0=$ no pain, $5=$ moderate pain, and $10=$ worst possible pain). Abbreviations: HUD, heads-up display; IQR, interquartile Range; n, number; SD, standard deviation.

estimated annual case volume, and self-reported average level of baseline back and neck pain $(P<0.02$; Table 1$)$. Neither age, years practicing ophthalmology, HUD use, or other pain characteristics differed between groups $(P>0.202)$. There was a significant difference in the distributions of anterior- and posterior-segment surgeon responses for the statements related to reduced severity
( $P=0.011)$ or frequency $(P=0.011)$ of pain and discomfort with HUD compared to a conventional microscope. Although there was a large positive response from posterior-segment surgeons for HUD reducing the severity (49\% agreement vs $14 \%$ disagreement) or frequency ( $49 \%$ vs $11 \%$ ) of pain (Figure 3), the positive response was even higher among anterior-segment surgeons for these benefits 
Table 2 Reasons Provided for Device Preference

\begin{tabular}{|l|l|l|}
\hline Reason for Preference $^{\text {a }}$ & Heads-Up Display (n=44) & Conventional Microscope (n=20) \\
\hline Visualization & $34(77 \%)$ & $\mathrm{II}(42 \%)$ \\
Ergonomics/comfort & $27(61 \%)$ & $3(12 \%)$ \\
Experience/familiarity & $0(0 \%)$ & $3(12 \%)$ \\
Device performance/reliability & $0(0 \%)$ & $3(12 \%)$ \\
Speed & $0(0 \%)$ & $4(15 \%)$ \\
Teaching & $6(30 \%)^{\mathrm{b}}$ & $0(0 \%)^{\mathrm{b}}$ \\
Digital integration & $2(5 \%)$ & $0(0 \%)$ \\
Patient benefit & $\mathrm{I}(2 \%)$ & $0(0 \%)$ \\
Preference varied by procedure & $0(0 \%)$ & $2(8 \%)$ \\
Other/general preference & $2(5 \%)$ & $0(0 \%)$ \\
\hline
\end{tabular}

Notes: ${ }^{a} \mathrm{~A}$ total of 64 surgeons responded to the forced-choice selection of preference for heads-up display or microscope. Frequency distributions are presented by preference using a denominator of 44 for heads-up display and 20 for microscope, and are not mutually exclusive: 32 surgeons provided one reason for preference, 29 provided two reasons for preference, and three surgeons provided three reasons for preference in the open-ended explanation for preference. All reasons for preference were captured; ' $T$ The proportion of respondents who reported working in a teaching facility or in a learning role (ie, fellows) was used as the denominator ( $\mathrm{n}=20$ for headsup display, $\mathrm{n}=\mathrm{I}$ I for conventional microscope).

( $84 \%$ vs $4 \%$ for severity and frequency questions) (Figure 2).

\section{Multivariable Regression}

A multivariable logistic regression analysis was performed to explore which variables were likely to be predictors of improvement in pain-related issues since introducing HUD. The model tested clinically relevant variables indicated by the primary investigators and included age, cumulative number of operating hours, type of surgeon, and proportion of cases completed with HUD. Stepwise selection for the best model resulted in two variables being retained, age and proportion of cases completed with HUD. The model indicated that surgeons who used HUD in $>50 \%$ of their cases had 5.12-times greater odds of reporting an improvement in pain since introducing the HUD ( $P=0.029$; Table 3 ). The model also suggested older age may have greater odds of reporting an improvement in pain-related issues; however, this did not reach statistical significance (1.05 greater odds, $P=0.152$; Table 3 ).

\section{Discussion}

This study used an observational cross-sectional survey design to understand use of HUD by US ophthalmic surgeons, and to compare the ergonomic outcomes associated with HUD and a conventional optical microscope in the OR. The response rate was smaller than other MSD surveys administered to ophthalmologists in the US, but higher than previously conducted studies of HUD in ophthalmology. 3,8

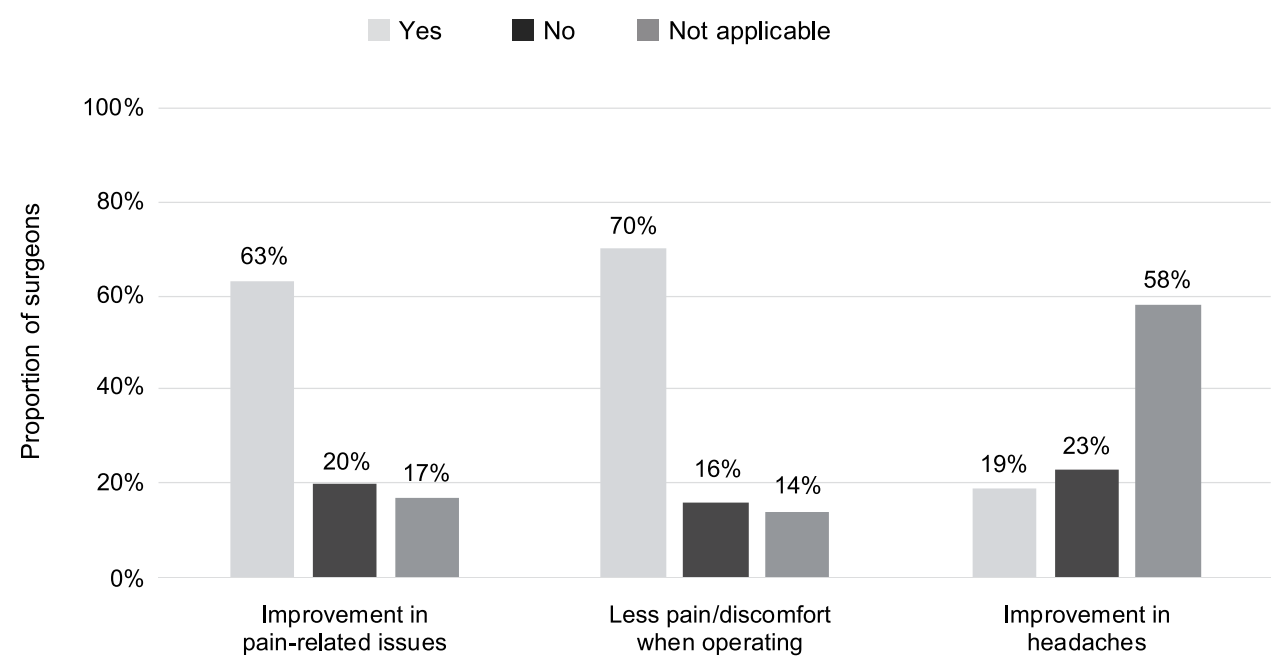

Figure I Reported changes in headache, pain/discomfort when operating, and pain-related issues after introducing heads-up display. 


\section{A Reduced the severity of pain and discomfort}

84

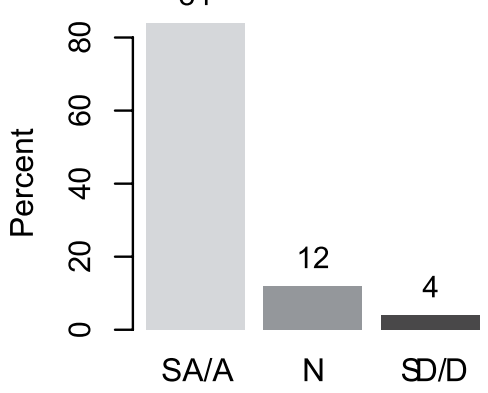

Reduced the frequency
of pain and discomfort

84

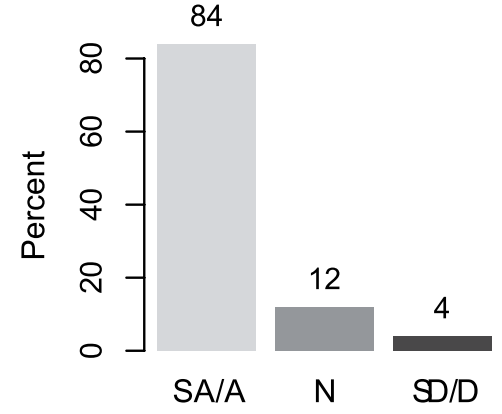

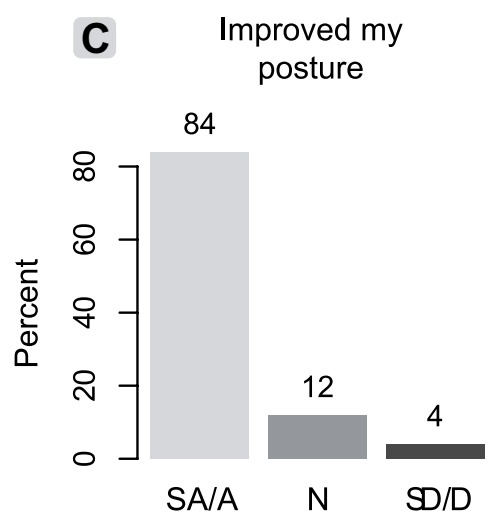

Improved my overall comfort

84



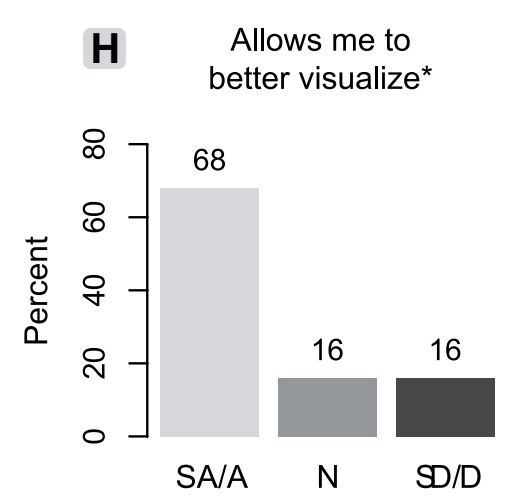
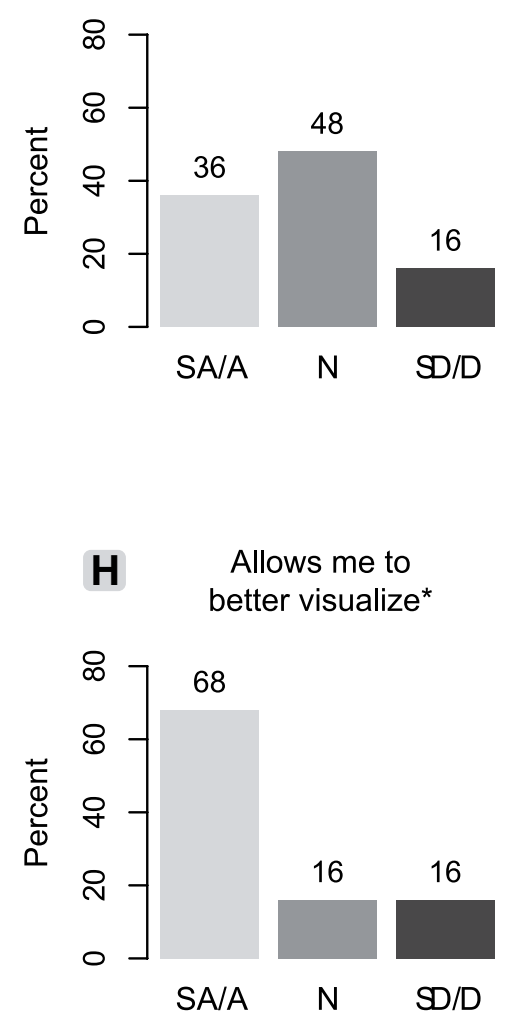

F Improved my physical performance

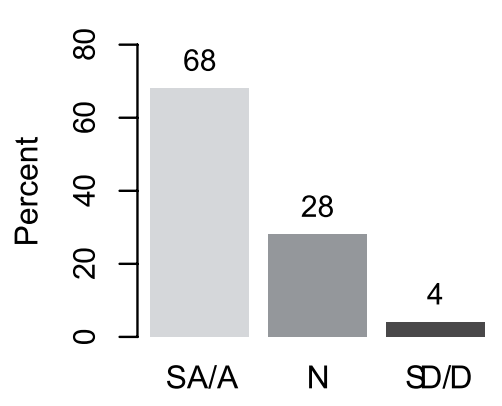



Figure 2 Frequency distribution of responses from 25 anterior-segment surgeons to the question: Compared to a conventional microscope, the use of a heads-up visualization system in the operating room: (A) Reduced the severity of pain and discomfort; (B) Reduced the frequency of pain and discomfort; (C) Improved my posture; (D) Improved my overall comfort; (E) Improved my mental performance; (F) Improved my physical performance; (G) Improved my confidence; (H) Allows me to better visualize; and (I) Allows me to operate more comfortably.**

Notes: *Allows me to better visualize the areas and angles required for surgery; **Allows me to operate more comfortably under higher magnification. Abbreviations: N, neutral; SA/A, strongly agree or agree; SD/D, strongly disagree or disagree.

Several pieces of data in this analysis substantiate the ergonomic benefit of operating heads-up. First, $82 \%$ of applicable surgeons reported feeling less pain/discomfort during operation since using HUD technology. Second, the logistic regression model found those who reported using the device in $>50 \%$ of cases had $>5$ times greater odds of reporting an improvement in pain since introducing HUD in the OR. Third, ergonomics was the second most common reason provided by surgeons to explain their preference for HUD. Posture has been identified as 

A Reduced the severity of pain and discomfort
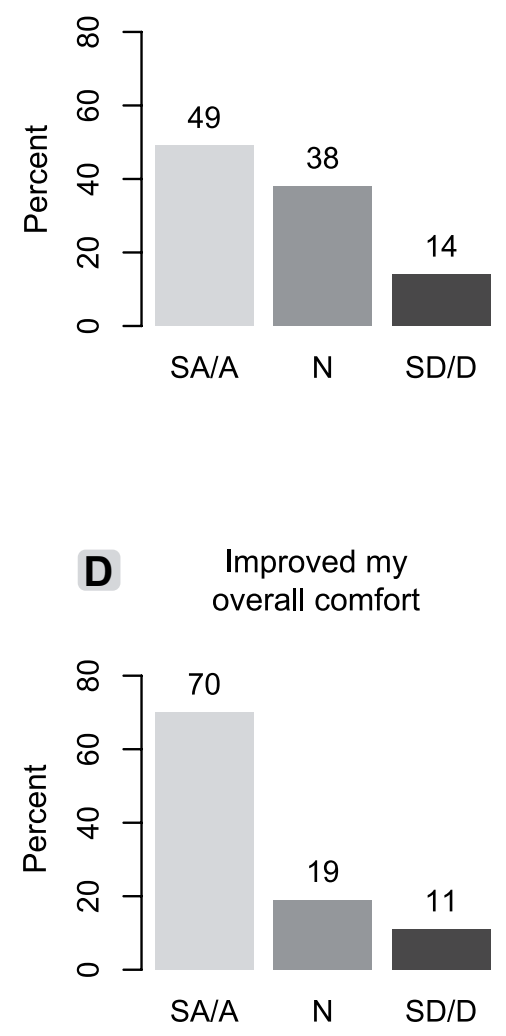

B Reduced the frequency
of pain and discomfort

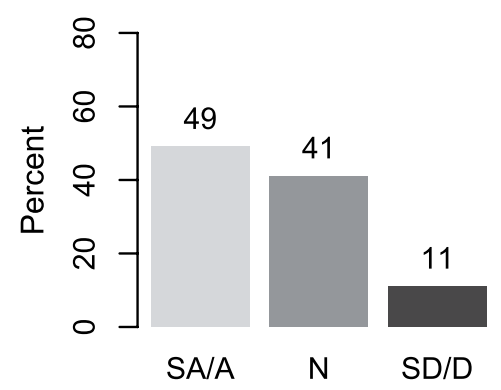

Improved my
posture

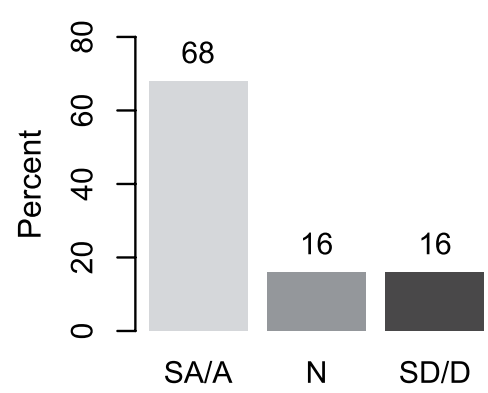

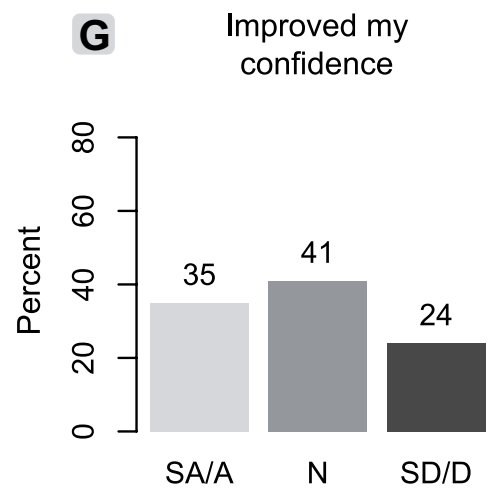
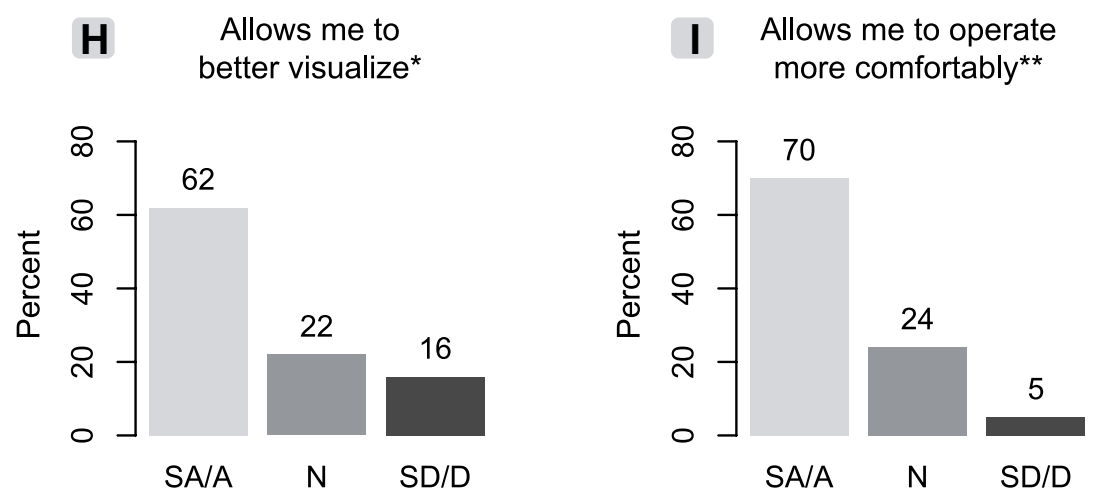

Figure 3 Frequency distribution of responses from 37 posterior-segment surgeons to the question: Compared to conventional microscope, the use of a heads-up visualization system in the operating room: (A) Reduced the severity of pain and discomfort; (B) Reduced the frequency of pain and discomfort; (C) Improved my posture; (D) Improved my overall comfort; (E) Improved my mental performance; (F) Improved my physical performance; (G) Improved my confidence; (H) Allows me to better visualize; $*$ and (I) Allows me to operate more comfortably.**

Notes: *Allows me to better visualize the areas and angles required for surgery; **Allows me to operate more comfortably under higher magnification.

Abbreviations: $\mathrm{N}$, neutral; SA/A, strongly agree or agree; SD/D, strongly disagree or disagree.

the most common cause of pain in surgeons. ${ }^{7}$ The US Occupational Safety and Health Administration notes that improvements in ergonomics can help to increase productivity, lessen muscle fatigue, and reduce the number and severity of work-related MSDs. Literature indicates operating heads-up can alleviate some joint pressure and muscle strain in the neck and back by minimizing nonneutral hyperextensions and flexions required for 
Table 3 Multivariable Logistic Regression Analysis for Predictors of Improvement in Pain-Related Issues Since Introducing Heads-Up Display

\begin{tabular}{|l|l|l|l|}
\hline Variables & Estimate (SE) & $\mathbf{Z}$ & $\boldsymbol{P}$-value \\
\hline Intercept & $-2.063(1.746)$ & -1.181 & 0.237 \\
Age (years) & $0.056(0.039)$ & 1.434 & 0.152 \\
Proportion of cases completed with heads-up display (>50\%)* & $1.635(0.749)$ & 2.180 & 0.029 \\
\hline
\end{tabular}

Note: *Referent category was $\leq 50 \%$ of cases.

microscope use. ${ }^{4,10,11}$ Objective analysis comparing these two modalities in the OR would be helpful for validation purposes.

The model suggested that surgeons of older age may have greater odds of reporting an improvement in painrelated issues. MSDs are progressive problems, and longer practice duration has been associated with more frequent back pain in ophthalmologists. ${ }^{17}$ Older surgeons who experience more MSDs may be better able to detect an improvement following an ergonomic intervention.

Approximately three-quarters of surgeons in this study agreed or strongly agreed that HUDs provided a benefit over microscope in improving posture and comfort, in line with previous studies in this space reporting similar statistics. ${ }^{13,14}$ The strong positive response regarding improved physical performance is also in line with other reports where ophthalmic participants noted reductions in physical fatigue. ${ }^{12}$ The finding that $39 \%$ of surgeons agreed that HUD improved mental performance compared to a conventional microscope is novel, as available published studies have not compared these devices on this parameter. Occupational health and wellness research indicates a connection between mental and physical stress. Mental demands such as procedure complexity, peer evaluation, multitasking, and distractions can contribute to stress within the OR. ${ }^{18}$ Dhmitri et $\mathrm{al}^{3}$ reported ophthalmologists' stress levels were independently associated with an increased prevalence of MSD in the neck, lower back, and upper extremities. Increasing levels of job strain were also associated with increased frequency of neck and low back pain in eye care providers and general practitioners. ${ }^{2}$ In turn, pain is frequently associated with cognitive impairment. ${ }^{19}$ Although this study is not designed to answer how HUDs might improve mental performance, it is possible that the pain reduction provided by the improved ergonomics of HUDs also provided a subsequent benefit in mental performance.

In the current study, $69 \%$ of the sample reported preference for the HUD, with $86 \%$ responding they would recommend it to their peers. This may be indicative of personal preference in surgery, in that surgeons who may not select it for themselves still recognize overall benefits and how it may be applicable to other surgeons. In addition, this is likely subject to potential significant bias in that most of these surgeons worked at institutions where the HUD had been purchased. It is likely that these systems would not have been purchased/installed without significant support/interest from surgeons. Data on preference for HUD is limited. There are other recently published studies where surgeons significantly favored the heads-up method compared to a conventional microscope ${ }^{20}$ or where $100 \%$ of surgical staff and students preferred HUD; ${ }^{12}$ however, these are pilot studies limited by very small sample sizes of less than 15 participants. Consistent with our sample, reasons for preferring HUD in the literature included comfort, visualization, and enhanced teaching. ${ }^{12-14}$ Reasons for preferring microscope somewhat aligned with criticisms of HUD technologies in pilot studies, such as situation-specific visualization (eg, when operating on an opacified capsule) or trouble adjusting to the screen. ${ }^{12}$ Benefits relating to "ease-of-use", surgical manipulations, and techniques cited in the literature $^{13}$ were notably absent in responses from study participants. This may be due to a high proportion of attending ophthalmologists and high mean device use in the study population, as respondents who are already very comfortable with surgical techniques and the device may notice different benefits, or think them less remarkable, than those perceived by residents or trainees.

Subgroup analysis revealed that anterior- and posteriorsegment surgeons had significantly different frequency distributions for whether heads-up display reduced the severity or frequency of pain and discomfort compared to a conventional microscope, with the proportion in agreement considerably higher among anterior-segment surgeons. Prolonged postures are known to result in cumulative strain. ${ }^{21}$ Given that anterior-segment surgeries are much shorter than posterior-segment surgeries, this finding 
of the difference in the perceptions of benefit was unexpected. However, another important contributor to MSDs is task repetition and intensity. ${ }^{21}$ Anterior-segment surgeons have a higher case load, meaning they experience more repetition in set-up, routine surgical tasks, and room turn-over than posterior-segment surgeons. The higher median baseline neck and back pain reported by anteriorsegment respondents in this sample may suggest the importance of repetition and intensity. Indeed, the number of surgeries per week has been reported as an important predictor of MSDs in open and laparoscopic surgeons. ${ }^{22,23}$ Anterior-segment surgeons may also experience more time pressure due to volume intensity. Surgeons working under time constraints often forgo breaks and concentrate with less regard for ergonomic adjustments while operating. ${ }^{9}$ Alternatively, as HUD technologies are more novel to anterior-segment surgeons, they may be more inclined to report improvement or better able to recall this improvement versus microscope compared to posterior-segment surgeons. Future studies should consider assessing job strains to contribute to the understanding of risk factors associated with each therapeutic area.

This study has several limitations. First, the majority of respondents were recruited from a device-install list which was considered necessary given the low market penetration of HUD systems in the US, predisposing the population to a disproportionate number of surgeons operating on the NGENUITY ${ }^{\circledR} 3 \mathrm{D}$ visualization system. However, the study cohort included sites with the TrueVision ${ }^{\circledR} 3 \mathrm{D}$ system and the Artevo system. As mentioned previously, the fact that these systems were installed suggests that these surgeons would have a favorable initial impression of the HUD. Second, only those with access to or experience on the device were eligible to participate, which prevented comparison to a control group, and limits generalizability to a larger population. This limitation was somewhat mitigated by the wide range of experience, surgical volume, and amount of use of the heads-up display, and the questionnaire design, which asked the surgeon to directly compare between devices, serving as a self-control, recalling back to a time when they only used a microscope. In addition, the response rate was higher than other studies on US ophthalmologists ${ }^{3,8}$ and represents a considerable portion of the device users in the US, which positively affects the study's internal validity. Although necessary given that many ophthalmic surgeons are unable to fully convert to heads-up display, the accuracy of recall may have been impacted by asking respondents to recall over a long period of time. Finally, the questionnaire-based design precludes statements on causality between an intervention and effect (as in all cross-sectional studies). Although it could not realistically capture all of the variables within the OR, or a surgeon's life, that could impact pain and MSDs, the questionnaire was developed based on an extensive literature review and clinical input from the principal investigators to reflect key factors in surgical ergonomics, balancing comprehensive variable capture without compromising completion of the survey.

\section{Conclusions}

In conclusion, this study compared surgeon-reported ergonomic benefits of HUD technology with a conventional microscope, and built on existing literature by assessing a larger sample size, including both anterior- and posterior-segment surgeons to be more reflective of the real world, and performing robust statistical analysis techniques. Promoting ergonomics is critical to ensure surgeon well-being and productivity. In this study, the majority of respondents reported an ergonomic benefit for HUD systems compared to conventional microscopes, including improved posture and comfort and reduced pain, as well as unexpected benefits for confidence and mental performance. Additional work is needed to promote awareness of ergonomics and prevention of MSDs, and educate users on the appropriate set-up of HUDs to achieve optimal ergonomics. HUD systems could be considered an important tool to improve the ergonomic environment of ophthalmic operating rooms. Additional studies with expanded enrollment to increase external validity, or those using a randomized prospective design would be helpful to provide additional information related to ergonomics and the potential benefit of HUD platforms.

\section{Abbreviations}

HUD, heads-up display; MSD, musculoskeletal disorder; OR, operating room; US, United States of America.

\section{Funding}

Alcon provided funding for the study.

\section{Disclosure}

LPM and HC are employees of Alcon. NCF, MHAG, and RAQ are employees of CRG-EVERSANA, which was contracted by Alcon to conduct the research. RJW is a consultant for Alcon, Bausch \& Lomb, Omeros, J\&J Vision, LENSAR, and EyePoint Pharmaceuticals; Speaker for Alcon, Bausch \& 
Lomb, Omeros, and Sun Pharma; Ownership interest in RxSight, RPS, EyeSafe, Pogotec, UBeam, and Tissuetech. JPE is a consultant for Aerpio, Regeneron, Alcon, Genentech/Roche, Novartis, Zeiss, Thrombogenics/Oxurion, Allergan, Allegro, Leica, Santen; Research support from Aerpio, Regeneron, Genentech, Alcon, Novartis, Thrombogenics/Oxurion, Allergan; Patent with Leica. The authors report no other conflicts of interest in this work.

\section{References}

1. Bureau of Labor Statistics. Nonfatal occupational injuries and illnesses requiring days away from work. 2015 Updated November 10, 2016. Available from: https://www.bls.gov/news.release/osh2.nr0. htm. Accessed July 27, 2020.

2. Kitzmann AS, Fethke NB, Baratz KH, Zimmerman MB, Hackbarth DJ, Gehrs KM. A survey study of musculoskeletal disorders among eye care physicians compared with family medicine physicians. Ophthalmology. 2012;119(2):213-220. doi:10.1016/j. ophtha.2011.06.034

3. Dhimitri KC, McGwin G, McNeal SF, et al. Symptoms of musculoskeletal disorders in ophthalmologists. Am J Ophthalmol. 2005;139 (1):179-181. doi:10.1016/j.ajo.2004.06.091

4. Hyer JN, Lee RM, Chowdhury HR, Smith HB, Dhital A, Khandwala M. National survey of back \& neck pain amongst consultant ophthalmologists in the United Kingdom. Int Ophthalmol. 2015;35(6):769-775. doi:10.1007/s10792-015-0036-Z

5. Ohlsson K, Attewell RG, Pålsson B, et al. Repetitive industrial work and neck and upper limb disorders in females. Am J Ind Med. 1995;27(5):731-747. doi:10.1002/ajim.4700270508

6. Capone AC, Parikh PM, Gatti ME, Davidson BJ, Davison SP. Occupational injury in plastic surgeons. Plast Reconstr Surg. 2010;125:1555-1561. doi:10.1097/PRS.0b013e3181d62a94

7. Soueid A, Oudit D, Thiagarajah S, et al. The pain of surgery: pain experienced by surgeons while operating. Int J Surg. 2010;8 (2):118-120. doi:10.1016/j.ijsu.2009.11.008

8. Sivak-Callcott JA, Diaz SR, Ducatman AM, Rosen CL, Nimbarte AD, Sedgeman JA. A survey study of occupational pain and injury in ophthalmic plastic surgeons. Ophthalmic Plast Reconstr Surg. 2011;27(1):28-32. doi:10.1097/IOP.0b013e3181e99cc8

9. Stucky CH, Cromwell KD, Voss RK, et al. Surgeon symptoms, strain, and selections: systematic review and meta-analysis of surgical ergonomics. Ann Med Surg (Lond). 2018;27:1-8. doi:10.1016/j. amsu.2017.12.013

10. Hallbeck MS, Lowndes BR, Bingener J, et al. The impact of intraoperative microbreaks with exercises on surgeons: a multi-center cohort study. Appl Ergon. 2017;60:334-341. doi:10.1016/j. apergo.2016.12.006

Clinical Ophthalmology

\section{Publish your work in this journal}

Clinical Ophthalmology is an international, peer-reviewed journal covering all subspecialties within ophthalmology. Key topics include: Optometry; Visual science; Pharmacology and drug therapy in eye diseases; Basic Sciences; Primary and Secondary eye care; Patient Safety and Quality of Care Improvements. This journal is indexed on PubMed

Submit your manuscript here: https://www.dovepress.com/clinical-ophthalmology-journal
11. van Det MJ, Meijerink WJ, Hoff C, van Veelen MA, Pierie JP. Ergonomic assessment of neck posture in the minimally invasive surgery suite during laparoscopic cholecystectomy. Surg Endosc. 2008;22(11):2421-2427. doi:10.1007/s00464-008-0042-6

12. Zhang Z, Wang L, Wei Y, Fang D, Fan S, Zhang S. The preliminary experiences with three-dimensional heads-up display viewing system for vitreoretinal surgery under various status. Curr Eye Res. 2019;44 (1):102-109. doi:10.1080/02713683.2018.1526305

13. Eckardt C, Paulo EB. Heads-up surgery for vitreoretinal procedures: an experimental and clinical study. Retina. 2016;36(1):137-147. doi:10.1097/IAE.0000000000000689

14. Mendez BM, Chiodo MV, Vandevender D, Patel PA. Heads-up 3D microscopy: an ergonomic and educational approach to microsurgery. Plast Reconstr Surg Glob Open. 2016;4(5):e717. doi:10.1097/ GOX.0000000000000727

15. Kuorinka I, Jonsson B, Kilbom A, et al. Standardised Nordic questionnaires for the analysis of musculoskeletal symptoms. Appl Ergon. 1987;18(3):233-237. doi:10.1016/0003-6870(87)90010-х

16. R Core Team. R: A language and environment for statistical computing. Vienna, Austria: R Foundation for Statistical Computing; 2019.

17. Chatterjee A, Ryan WG, Rosen ES. Back pain in ophthalmologists. Eye (Lond). 1994;8(Pt 4):473-474. doi:10.1038/eye.1994.112

18. Wilson MR, Poolton JM, Malhotra N, Ngo K, Bright E, Masters RS. Development and validation of a surgical workload measure: the surgery task load index (SURG-TLX). World J Surg. 2011;35 (9):1961-1969. doi:10.1007/s00268-011-1141-4

19. Nadar MS, Jasem Z, Manee FS. The cognitive functions in adults with chronic pain: a comparative study. Pain Res Manag. 2016;2016:5719380. doi:10.1155/2016/5719380

20. Palácios RM, de Carvalho ACM, Maia M, Caiado RR, Camilo DAG, Farah ME. An experimental and clinical study on the initial experiences of Brazilian vitreoretinal surgeons with heads-up surgery. Graefes Arch Clin Exp Ophthalmol. 2019;257(3):473-483. doi:10.1007/s00417-019-04246-w

21. McAtamney L, Nigel Corlett E. RULA: a survey method for the investigation of work-related upper limb disorders. Appl Ergon. 1993;24(2):91-99. doi:10.1016/0003-6870(93)90080-s

22. Plerhoples TA, Hernandez-Boussard T, Wren SM. The aching surgeon: a survey of physical discomfort and symptoms following open, laparoscopic, and robotic surgery. J Robot Surg. 2012;6(1):65-72. doi:10.1007/s11701-011-0330-3

23. Szeto GP, Ho P, Ting AC, Poon JT, Cheng SW, Tsang RC. Workrelated musculoskeletal symptoms in surgeons. J Occup Rehabil. 2009;19(2):175-184. doi:10.1007/s10926-009-9176-1
Central and CAS, and is the official journal of The Society of Clinical Ophthalmology (SCO). The manuscript management system is completely online and includes a very quick and fair peer-review system, which is all easy to use. Visit http://www.dovepress.com/ testimonials.php to read real quotes from published authors. 